\title{
3D printing nanocomposite hydrogels with lattice vascular networks using stereolithography
}

\author{
Livia M. Kalossaka ${ }^{1,2,3, b)}$ (D, Ali A. Mohammed ${ }^{1, b)}$, Giovanni Sena ${ }^{2}$, Laura Barter ${ }^{3,4}$, \\ Connor Myant ${ }^{1, a)}$ \\ ${ }^{1}$ Dyson School of Design Engineering, Imperial College London, London SW7 2AZ, UK \\ ${ }^{2}$ Department of Life Sciences, Imperial College London, London SW7 2AZ, UK \\ ${ }^{3}$ Department of Chemistry, Molecular Sciences Research Hub, Imperial College London, 82 Wood Lane, London W12 0BZ, UK \\ ${ }_{4}^{4}$ Institute of Chemical Biology, Molecular Sciences Research Hub, Imperial College London, 82 Wood Lane, London W12 0BZ, UK \\ a) Address all correspondence to this author. e-mail: connor.myant@imperial.ac.uk \\ b) These authors share first co-authorship.
}

Received: 9 July 2021; accepted: 7 October 2021; published online: 28 October 2021

\begin{abstract}
Hydrogels have emerged as leading candidates to reproduce native extracellular matrix. To provide structures and functions similar to tissues in vivo, controlled porosity and vascular networks are required. However, fabrication techniques to introduce these are still limited. In this study we propose stereolithography as a fabrication technique to achieve 3D vascular networks using water-based solvents only. A 3D printable hydrogel is formulated based on available commercial chemicals such as acrylamide (AAm) and polyethylene glycol diacrylate 700 (PEGDA700), with nanocellulose crystals (CNC) as a nanofiller. An optimisation procedure to increase resolution, tune porosity as well as mechanical properties is developed. The results highlight the importance of photoabsorber addition to improve channel resolution. We demonstrate that with the adequate choice of chemicals and fillers for photocurable formulations, structural and functional properties of the fabricated scaffold can be tailored, opening the path for advanced applications.
\end{abstract}

\section{Introduction}

Hydrogels are hydrophilic 3D polymeric networks that are cross-linked together using ionic, covalent, or physical crosslinks with the capability of holding large volumes of water. These hydrophilic materials possess numerous unique properties such as flexibility, transparency, permeability, biocompatibility, and low friction $[1,2]$. Natural, synthetic and hybrid polymer formulations allow for a wide range of hydrogels with varying biochemical and mechanical properties to be formed $[3,4]$. These often consist of hydrophilic chemical groups such as $-\mathrm{NH}_{2}$, $-\mathrm{COOH},-\mathrm{OH},-\mathrm{CONH}_{2},-\mathrm{CONH}$, and $-\mathrm{SO}_{3} \mathrm{H}[5,6]$.

Single network hydrogels, usually consisting of a crosslinked hydrophilic homopolymer, are soft, weak and brittle. Their tensile and compressive properties are normally in the sub-MPa range and their ability to withstand strains are commonly less than $100 \%$. However, load-bearing and naturally occurring soft tissues are much tougher, stronger and can handle strains greater than $100 \%$. Developments in hydrogel technology have moved past single network systems due to their physical and biochemical limitations [7]. To develop novel alternatives for existing biomaterials, double network (DN) hydrogels have been created using two contrasting polymer networks resulting in a synergetic effect capable of replicating the physical properties of native human tissue such as cartilage and bone [8-10]. Nanocomposite structures such as silica nanoparticles (SNPs) [11], copper nanopowder [12, 13], laponite clay [13], nanoceria (NC) $[14]$ and nanocellulose crystals (CNC) $[15,16]$ have been used to further enhance the material properties of hydrogels. The integration of nanocomposite structures results in internal physical reinforcement to external forces, enhanced cellular attachment due to changes in topography and a potential for electrical signals and sensing $[8,11,17,18]$.

The polymer networks that make up hydrogels are often created using conventional fabrication techniques such as thermal and photo polymerisation $[19,20]$. These polymerisation processes often require degassing of oxygen to ensure no unreacted toxic monomers are left in the bulk of the material [11]. Therefore, material synthesis often takes place in closed systems. 
Closed systems limit the complexity of the physical design of the hydrogel, resulting in primitive shapes such as cylinders or cubes. Conventional manufacturing approaches, such as moulding, lack geometric flexibility and require extended curing time for bulk material synthesis. Moulding also limits the possibility to customise each sample rapidly, as each mould requires time and is often expensive.

However, real-world applications such as tissue engineered scaffolds, microelectronics and substrates for soilless cultivation require complex architectural designs [21-23]. For example, hydrogels can benefit from complex structures such as vascular and tubular networks for nutrient distribution, blood vessel penetration and cellular growth in tissue engineering $[4,7]$, integrated root growth through complex channels in substrates for soilless cultivation [23], capillary structures for smart electronics and MEMS devices [24-27], or customisable wearable technologies with sensing capabilities in smart textiles [28, 29]. The limitations faced in bulk synthesis with regards to shape and design complexity in hydrogels can be overcome using advanced techniques such as $3 \mathrm{D}$ printing.

3D printing eliminates the need for closed system material synthesis used in conventional manufacturing. Closed systems refer to fabrication processes whereby a material is created in an enclosed mould or cast that determines its final shape, whilst $3 \mathrm{D}$ printing builds on an open platform. 3D printing can significantly reduce the production costs, whilst speeding up testing processes and allowing for customisable solutions. Stereolithography (SLA), which is a light-based 3D printing technique, has become one of the most versatile and commonly used technologies. A photocurable resin is placed in a printing tank with a transparent base, such as PDMS, that is exposed to a UV-light laser [30]. This causes the resin to be photopolymerised against a build platform that moves in the $\mathrm{z}$-axis in a precise and controlled manner. Current commercially available SLA printers have the capability of moving within accuracies of 5-200 $\mu \mathrm{m}$ in the $z$-axis, with current laser-spot size as small as $85 \mu \mathrm{m}$ and $X Y$ resolution as accurate as $25 \mu \mathrm{m}$.

$3 \mathrm{D}$ printing formulations commonly consist of monomers that build the backbone of the material, cross-linkers that allow the material to solidify, photoinitiators that interact with light to initiate the reaction, and when required, photoabsorbers to limit the penetration of light to achieve a higher resolution [31]. However, limited success has been achieved in printing hydrogels with intricate and complex internal structures for applications that require a vascular network. This is due to the large water content relative to the reactive monomers, meaning the layer-bylayer build is compromised due to insufficient adhesion issues on the build plate, as well as difficulty in forming a structure that is able to support itself against gravity. Further, light penetration and depth whilst printing needs to be finely tuned to achieve the complex structures required by design. Therefore, effective photopolymerisation using 3D printing is highly dependent on carefully designed resin formulation. These formulations must be able to handle fast curing times that allow for continuous layer-by-layer builds, whilst maintaining a high resolution with low compound error throughout each layer [31].

Recent studies have shown nanocomposite structures can enhance mechanical properties of hydrogels after a specific threshold of loading has been reached within the material [32]. This is due to either covalent bonding points between the nanostructure and the polymer network, or as physical reinforcement to withstand damage against strain $[26,27]$. A recent study has shown that nanocellulose crystals (CNC) has the ability to enhance the mechanical properties of a PEG based photocurable resin using SLA [26]. CNC provides valuable material properties such as biocompatibility, hydrophilicity, cellular attachment points, and modifiable stiffness [26, 33, 34]. Previous research has shown consistent mechanical properties with the addition of $\mathrm{CNC}$ at concentrations ranging from 0 to $2.4 \mathrm{wt} \%$ [35].

This study provides evidence that printing high-water content hydrogels with complex interconnected internal structures is feasible using SLA. The monomers used to create the hydrogel structures in this work will be acrylamide (AAm) and polyethylene glycol diacrylate $700\left(\mathrm{PEGDA}_{700}\right)$, with CNC as a nanofiller. The choice of monomers was made according to previous studies [36]. Three structures (Solid, Hole and Lattice designs as shown in Supplementary Information 2) will be designed to determine the resolution, swelling and mechanical properties of the printed hydrogels. The study aims to show that high-water content hydrogels can be printed with high resolution and complex lattice structures, and that their mechanical properties can be tuned based on CNC content, as well as design.

\section{Results and discussion}

Acrylamide/PEGDA ${ }_{700}$ hydrogels loaded with $0 \%, 0.2 \%$ and $1 \%$ cellulose nanocrystals (CNC) were successfully printed using a Form2 3D printer (Formlabs, USA) as represented in Fig. 1. For all three compositions, CAD designs of $1 \mathrm{~cm} \times 1 \mathrm{~cm} \times 1 \mathrm{~cm}$ cubes with Solid, Hole and Lattice structures were printed. All structures were printed on the same platform, as shown in Supplementary Information 1 . Prior to achieving full 3D prints, an optimisation process was used to determine the optimum concentrations for each formulation. This was applied to achieve a printing resolution capable of creating the complex lattice structures.

\section{Optimisation process}

There has been much discussion about 3D printing optimisation for hydrogels. Successful biofabrication of hydrogels depends on both geometric accuracy and cell viability. These are dependent 


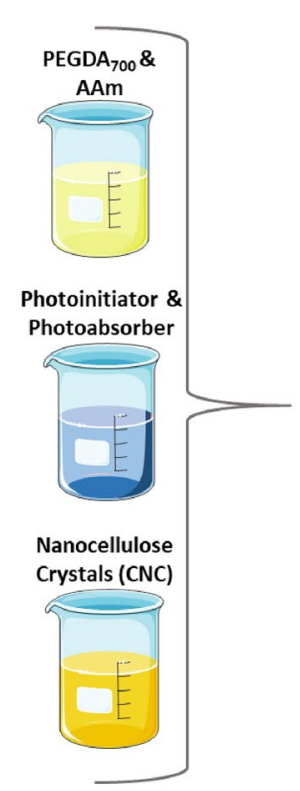

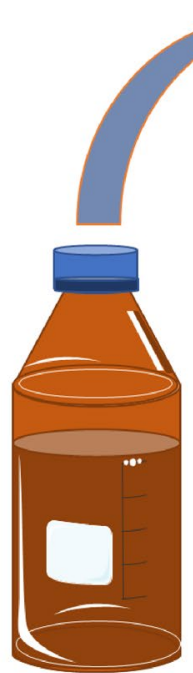

3D Printing Resin
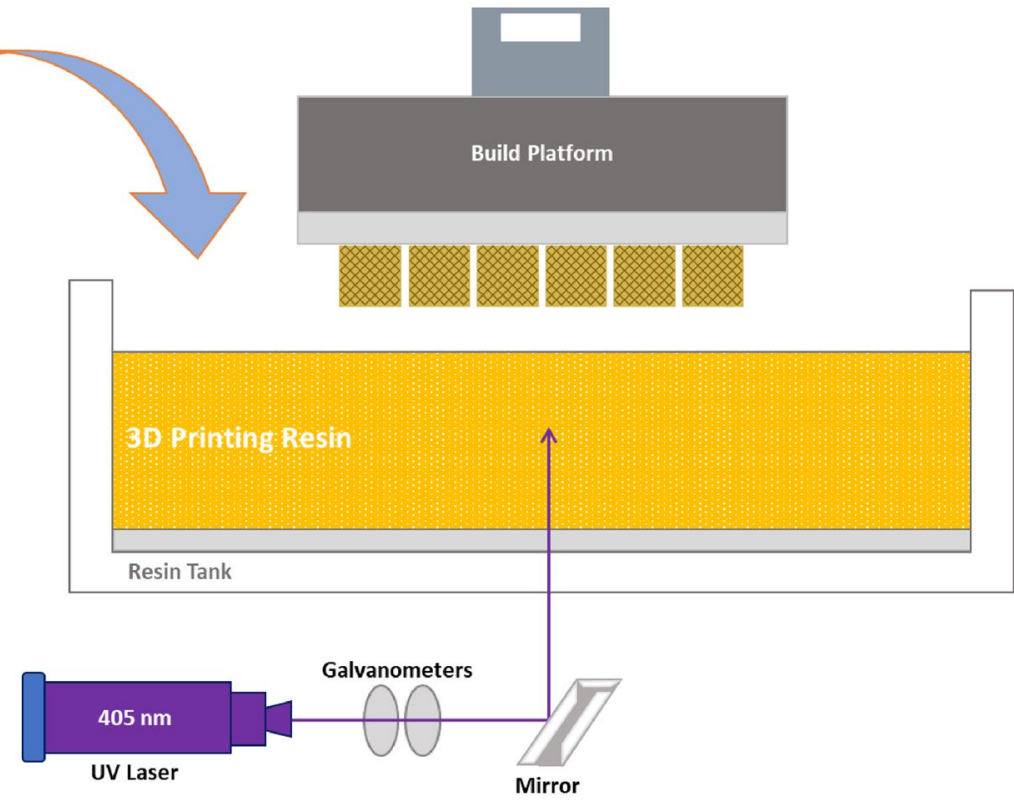

Figure 1: Schematic representation of a 3D printing process using a PEGDA 700 /AAm formulation with CNC precursors as nanocomposite fillers.

on the different printing parameters available (e.g. layer height, orientation), as well as the chemistry of the printing resin. The majority of biofabrication strategies revolve around material extrusion printing, hence research efforts have been directed to provide printing optimisation processes for this fabrication technique [37]. With regards to stereolithography, printing accuracy has been defined in several papers by trueness and precision
$[38,39]$. However, these studies are not specific to hydrogel fabrication. In this section, we will describe our novel printing optimisation process to achieve a successful hydrogel print, as presented in Fig. 2.

The first step during resin optimisation is to prepare a base formulation consisting of the chosen monomers and crosslinkers, a photoinitiator and a solvent. In this work, acrylamide

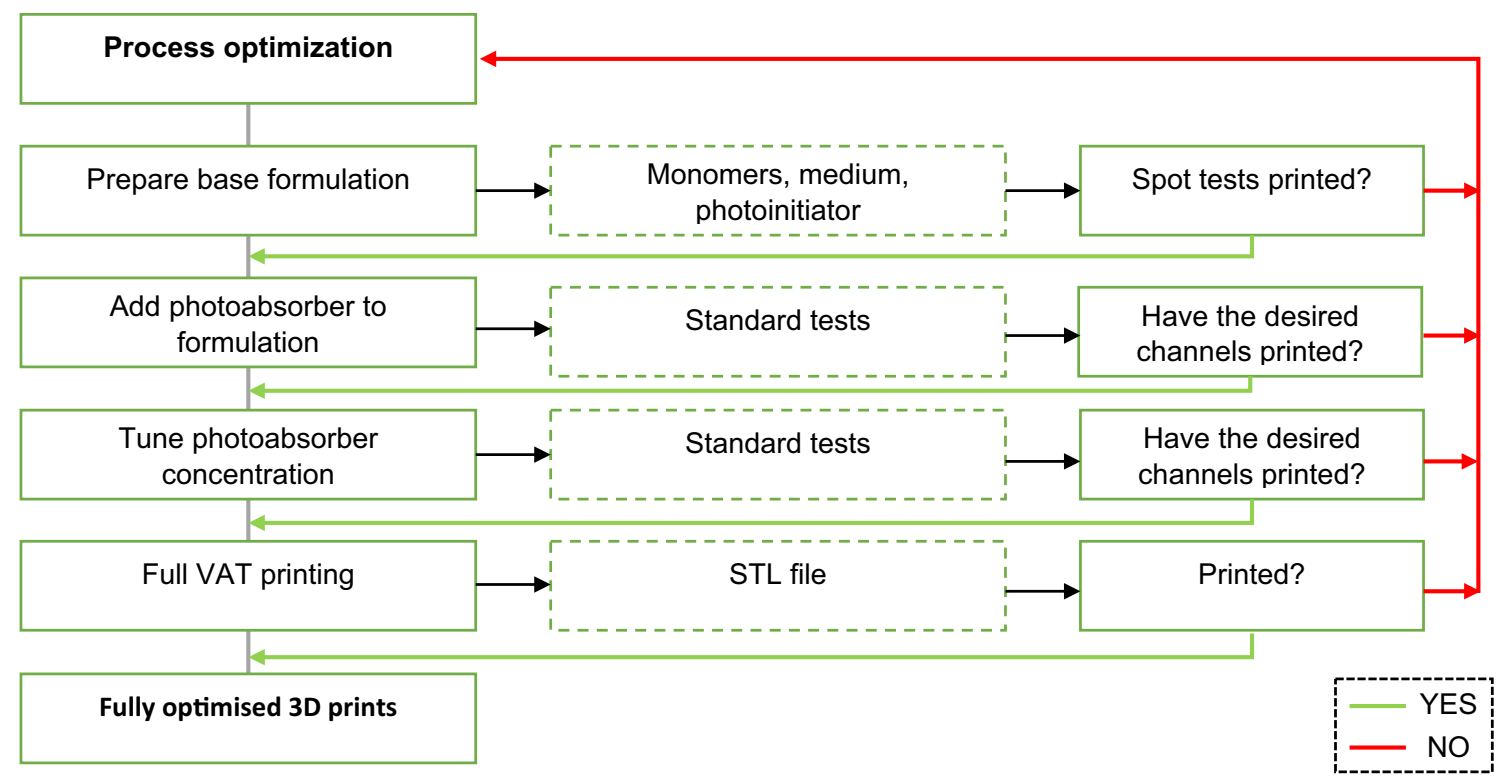

Figure 2: Process optimisation route for SLA printing hydrogels. 
(AAm) was the chosen monomer, poly (ethylene glycol) diacrylate $\left(\right.$ PEGDA $\left._{700}\right)$ as a cross-linker, lithium phenyl-2,4,6trimethylbenzoylphosphinate (LAP) as the photoinitiator and deionised water (DI-water) as the solvent. Once the base formulation is prepared, it is subjected to a spot test. A spot test is done by removing the build plate from the printer and allowing the laser trace to shine on the tank where the resin is placed. In this case, a disc $1 \mathrm{~cm}$ in diameter is left to trace for 1 layer. Once the layer is complete, the resin is examined to check if the formulation polymerises and forms a solid disc. If unsuccessful, the concentration of monomers and/or photoinitiator system is increased incrementally. Once a successful spot test is achieved, the disc diameter is measured and compared to the CAD file to determine whether there is any overcuring. Overcuring is the process in which light propagates longer than the desired time of exposure, and outside of the desired region of the CAD design.

If any overcuring occurs, a photoabsorber 1-phenylazo2-naphthol-6,8-disulfonic acid disodium salt (synonym; Orange G) is added to the formulation. Photoabsorbers limit the penetration depth of light, resulting in higher accuracy prints relative to the original CAD design. The tests developed in this study using the disc and minimum channel design (See Supplementary Informations 3 and 4) are used to evaluate the trueness and precision of the print. For statistical evidence, three standard tests are printed each time. After post-curing, the disc is visually inspected and if overcuring is observed, the photoabsorber concentration can be increased to limit the penetration depth.

Once the formulation is optimised, a full VAT of the desired model is printed. Depending on the model overhangs, printing resolution can be improved by choosing a smaller layer height. The structure is then examined using optical microscopy and Image J for analysis. Blue dye can be added to the printed structure to help increase contrast whilst using the optical microscope. If overcuring is still occurring on specific parts of the desired print, then a higher concentration of photoabsorber is added and hydrogels are reprinted. This process is repeated until the prints are formed within acceptable tolerances to their CAD design. It is important to note that a threshold of photoabsorber can be reached where light penetration is no longer sufficient to build layers, and causes the print to fail, this is known as undercuring.

\section{Minimum channel diameter analysis: Parameter optimisation}

Based on a total mass of $100 \mathrm{~g}$ DI-water, a resin formulation consisting of $15 \mathrm{w} / \mathrm{w} \%$ AAm and $5 \mathrm{w} / \mathrm{w} \% \mathrm{PEGDA}_{700}$ was mixed. Relative to the mass of AAm and PEGDA ${ }_{700}, 0.2 \%$ CNC, $1 \mathrm{w} / \mathrm{w} \%$ photoinitiator (LAP) and an initial $0.04 \mathrm{w} / \mathrm{w} \%$ concentration of photoabsorber Orange $\mathrm{G}$ were added as described in the experimental section.
Prints were placed under a microscope to evaluate the minimum channel width that is feasible using optimised formulations using $0.2 \%$ and $0.4 \%$ Orange G (Fig. 3). A CAD file was created using Autodesk Fusion 360, with dimensions of $20 \times 6 \mathrm{~mm}$ in $x / y$ and $5 \mathrm{~mm}$ in $z$ height. Square channels of varying width were constructed as reported in Fig. 3. Microscope analysis shows that trueness improves as the measured values become closer to the intended value for the formulation with $0.4 \%$ photoabsorber (Fig. 3e). Considering that the minimum spot size for Formlabs 2 is $140 \mu \mathrm{m}$, the minimum channel width printed is $280 \mu \mathrm{m}$ using the optimised formulation. The measured interval starts to deviate significantly from the intended size below $500 \mu \mathrm{m}$. This means that our formulation produced for VAT stereolithography can reliably produce channels with a width of $500 \mu \mathrm{m}$. Figure 3 demonstrates the change in resolution that occurs when increasing the photoabsorber concentration from 0.2 to $0.4 \%$.

\section{Swelling}

3D printed hydrogels across all three CNC concentrations were able to sustain the uptake of water without deformation or breakage, demonstrating viable mechanical integrity under hydrostatic pressures. This is a significant property for hydrogels as it suggests the shape of the prints can be maintained for their specific application, whilst swelling in hydrophilic mediums. Swelling studies were performed on the printed hydrogels to evaluate whether there is a difference in swelling ratios across print designs and CNC concentrations, presented in Fig. 4. Swelling Ratio Percentages (SR\%) were measured according to the formula below:

$$
\operatorname{SR}(\%)=\frac{W_{t f}-W_{t 0}}{W_{t 0}} \times 100
$$

where $W_{t f}$ represent the weight of the swollen sample after $48 \mathrm{~h}$ and $W_{t 0}$ is the weight of the sample before swelling. The swelling ratio of $0 \% \mathrm{CNC}$ was the largest across the three concentrations, whilst $1 \% \mathrm{CNC}$ showed the lowest swelling ratio. The results suggest that as the concentration of $\mathrm{CNC}$ increases in the resin, the swelling ratio decreases. This is potentially due to $\mathrm{CNC}$ acting as a filler and creating a denser, more compact network within the hydrogel, resulting in lower water uptake. In addition, $\mathrm{CNC}$ is hydrophilic, hence hydrogen bonding between the filler and the polymer chains may occur, decreasing the availability for hydrogen bonding with water. As the concentration of $\mathrm{CNC}$ increases, this effect becomes more pronounced as a result of stronger interfacial interaction between the PEGDA, AAm and CNC. Consequently, water molecules are hindered from penetrating into the cross-linked polymeric network [40]. The nanocomposite structure of the prints reduces the free volume available for water. Swelling ratios for Solid structures without 


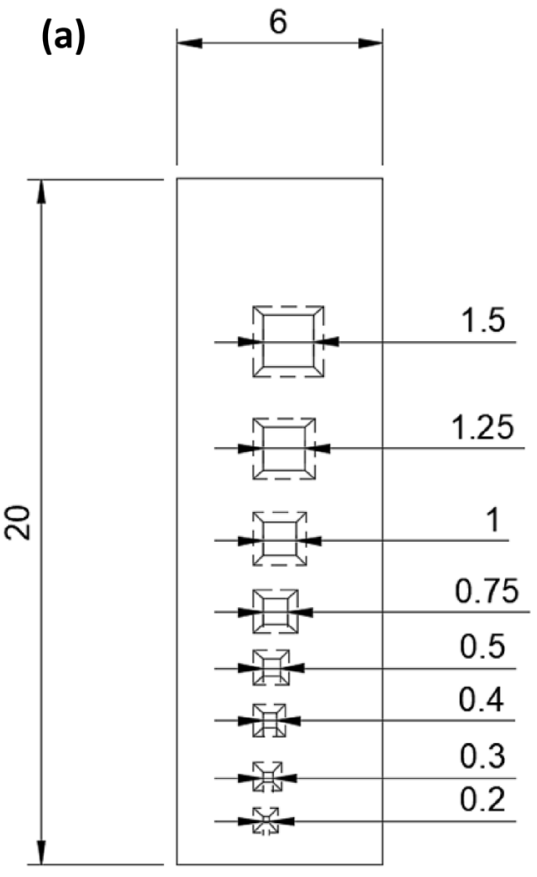

(d)

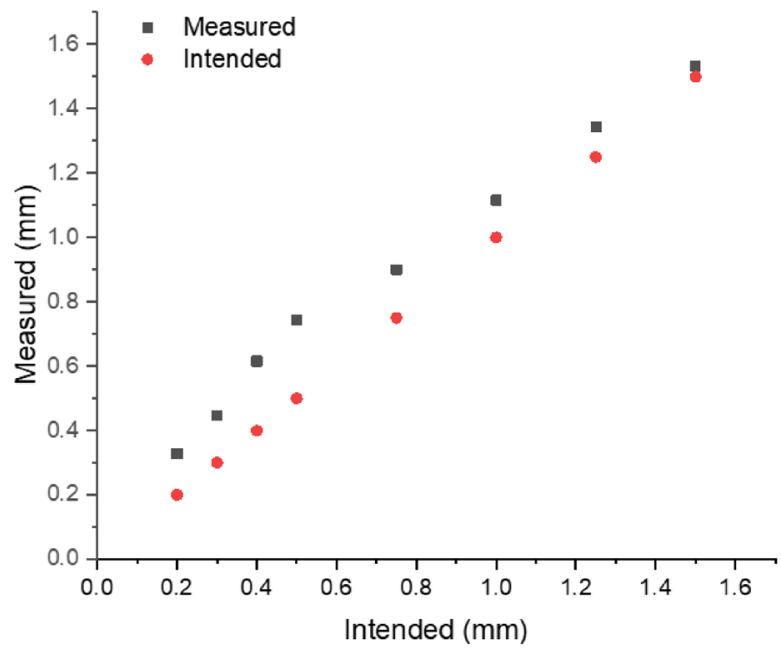

(b)

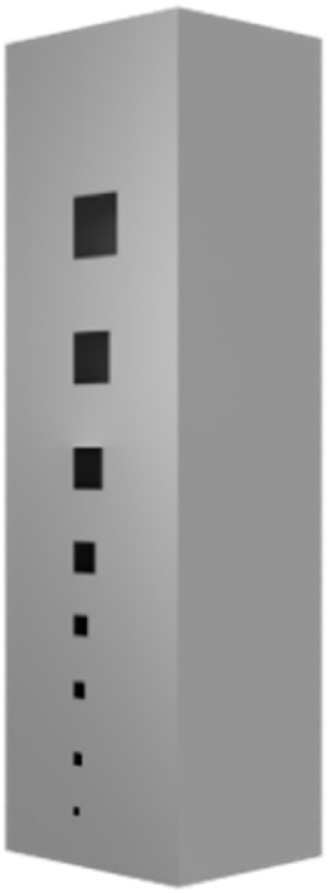

(c)

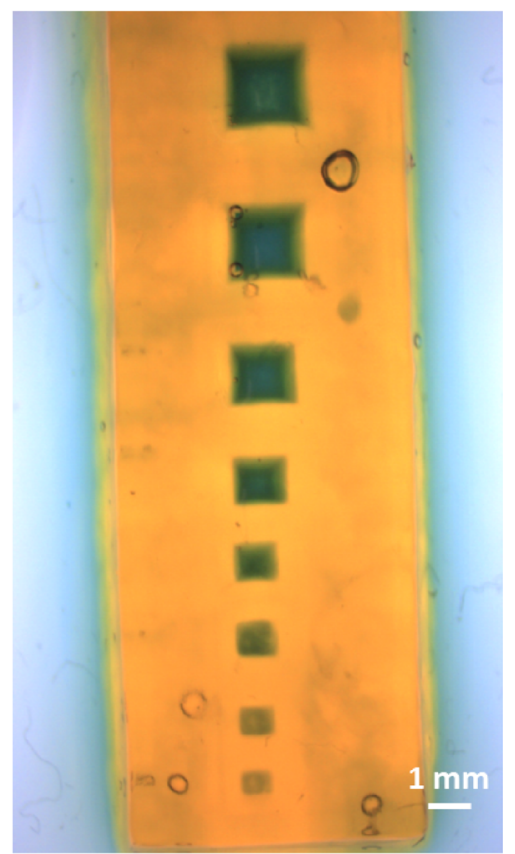

(e)

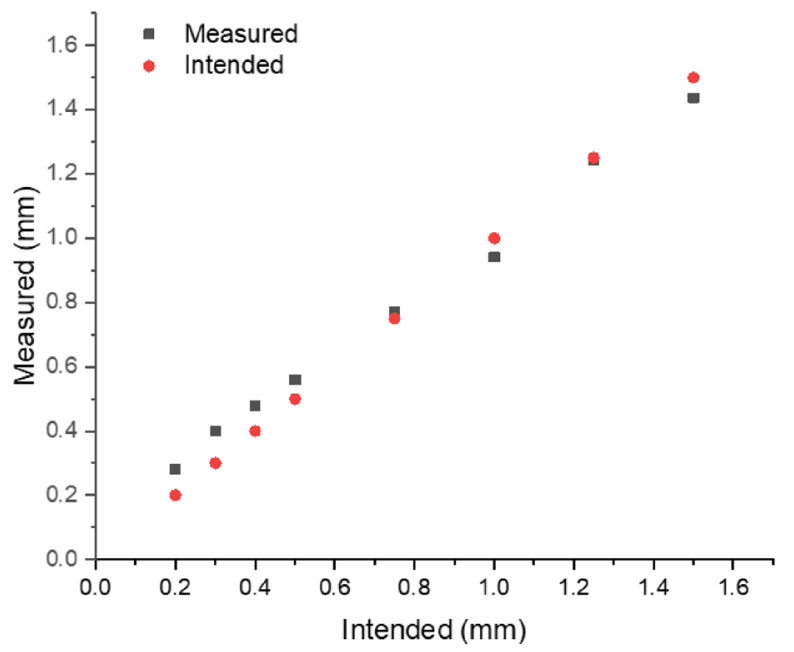

Figure 3: Fully optimised prints. (a) CAD file specifying the dimensions of the design structure used to study'measured vs. intended' dimensions in mm, (b) image of STL file used for printing, and (c) printed hydrogel with $0.4 \%$ photoabsorber Orange $\mathrm{G}$ imaged using blue dye to highlight all channels are visible. Graphs showing the Measured (3D printed) vs. Intended (CAD) sizes of channels printed using (d) $0.2 \%$ Orange G and (e) $0.4 \%$ Orange G.

CNC reached $42 \%$. The addition of $0.2 \% \mathrm{CNC}$ reduced the swelling ratio for Solid structures to $32 \%$, and further down for Solid structures $25 \%$ with $1 \%$ CNC. A similar trend was seen for the lattice structures.

The impact of water uptake and swelling on the dimensions of the 3D printed hydrogels was examined using an optical microscope. The Lattice 3D printed hydrogels were imaged under blue dye to increase contrast, as visualised in Fig. 5. Representative images showing changes in the width of the central channel were studied. The results are summarised in Table 1. $0 \%$ CNC resulted in $17.2 \%$ change in channel diameter after swelling, whereas $0.2 \% \mathrm{CNC}$ exhibited a $12.1 \%$ increase. Increasing $\mathrm{CNC}$ to $1 \%$ resulted in only a $0.8 \%$ increase in diameter. This suggests that a concentration as low as $1 \% \mathrm{CNC}$ is effective at reducing changes in the structure whilst still allowing the material to absorb $<30 \%$ water.

The channel width increases in diameter as swelling occurs; however, the mechanical integrity of the $3 \mathrm{D}$ printed hydrogels 

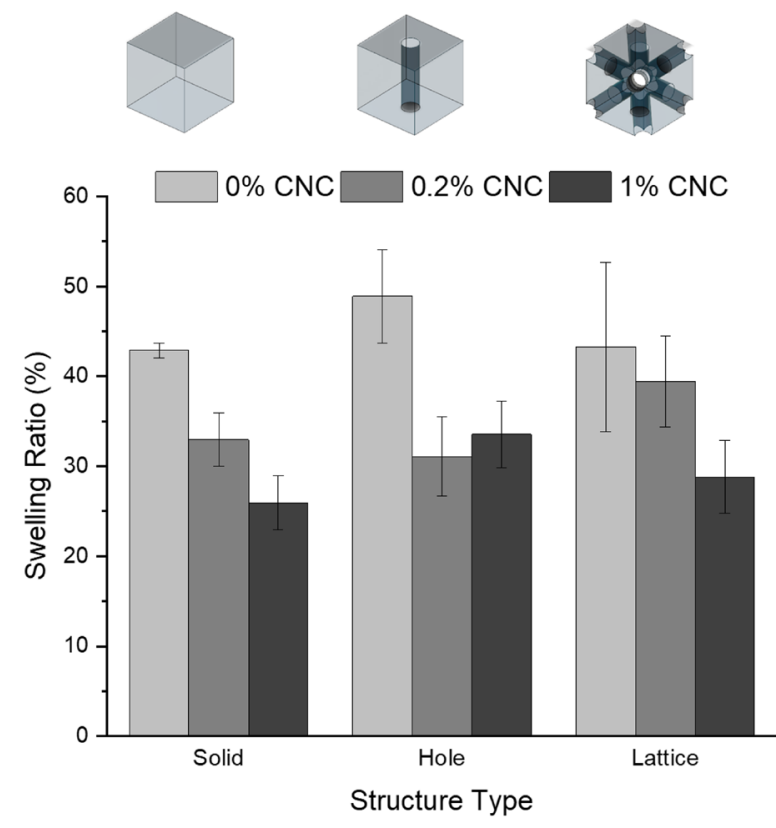

Figure 4: $C A D$ designs of the Hole, Lattice and Solid structures used to $3 D$ print the hydrogels and graph showing swelling ratios of $0 \% \mathrm{CNC}, 0.2 \%$ CNC and $1 \%$ CNC hydrogels with Solid, Hole and Lattice structures after $48 \mathrm{~h}$ in water.

are preserved, suggesting cross-links between the polymer chains are stable and sufficient to sustain hydrostatic pressures. The increase in channel width is more distinct in the samples with $0 \%$ CNC. As described previously the incorporation of nanofillers results in stronger adhesive forces which limit the impact of water swelling. The increased concentration of CNC within the prints, and ultimately within the polymer networks, reduces the free space for water uptake due to tighter crosslinking, which limits the hydrogel from expanding as freely [41].

\section{Fourier-transform infrared spectroscopy (FTIR) analysis}

FTIR spectroscopy was used to investigate the presence of unreacted monomers in the printed hydrogels. The results of the FTIR study are shown in Fig. 6, and the summary of the bands is presented in Table 2. Samples were compared directly after printing (Fresh), after being washed in water (Wash only), and after being washed and post-cured (Wash and Post-cure). The FTIR spectra of the $3 \mathrm{D}$ printed specimens shows the presence of a broad band at $3200 \mathrm{~cm}^{-1}$ representing water bonding to the polymer network. The peaks between 2900 and $2850 \mathrm{~cm}^{-1}$ represent the aliphatic $\mathrm{C}-\mathrm{H}$ stretching that are characteristic of the polymer backbone. In addition, the typical double bond $\mathrm{C}=\mathrm{O}$ peak around $1654 \mathrm{~cm}^{-1}$ and the $\mathrm{C}=\mathrm{O}$ group at $1717 \mathrm{~cm}^{-1}$ are present across all spectra. As the samples are washed and post-cured it can be observed that the unreacted monomer containing $\mathrm{C}-\mathrm{H}$ bonds, initially present at $938 \mathrm{~cm}^{-1}$, disappears completely after samples are washed and post-cured. Washing the prints by allowing them to swell in water allows the unreacted monomers or cross-linkers to leach out. The monomers

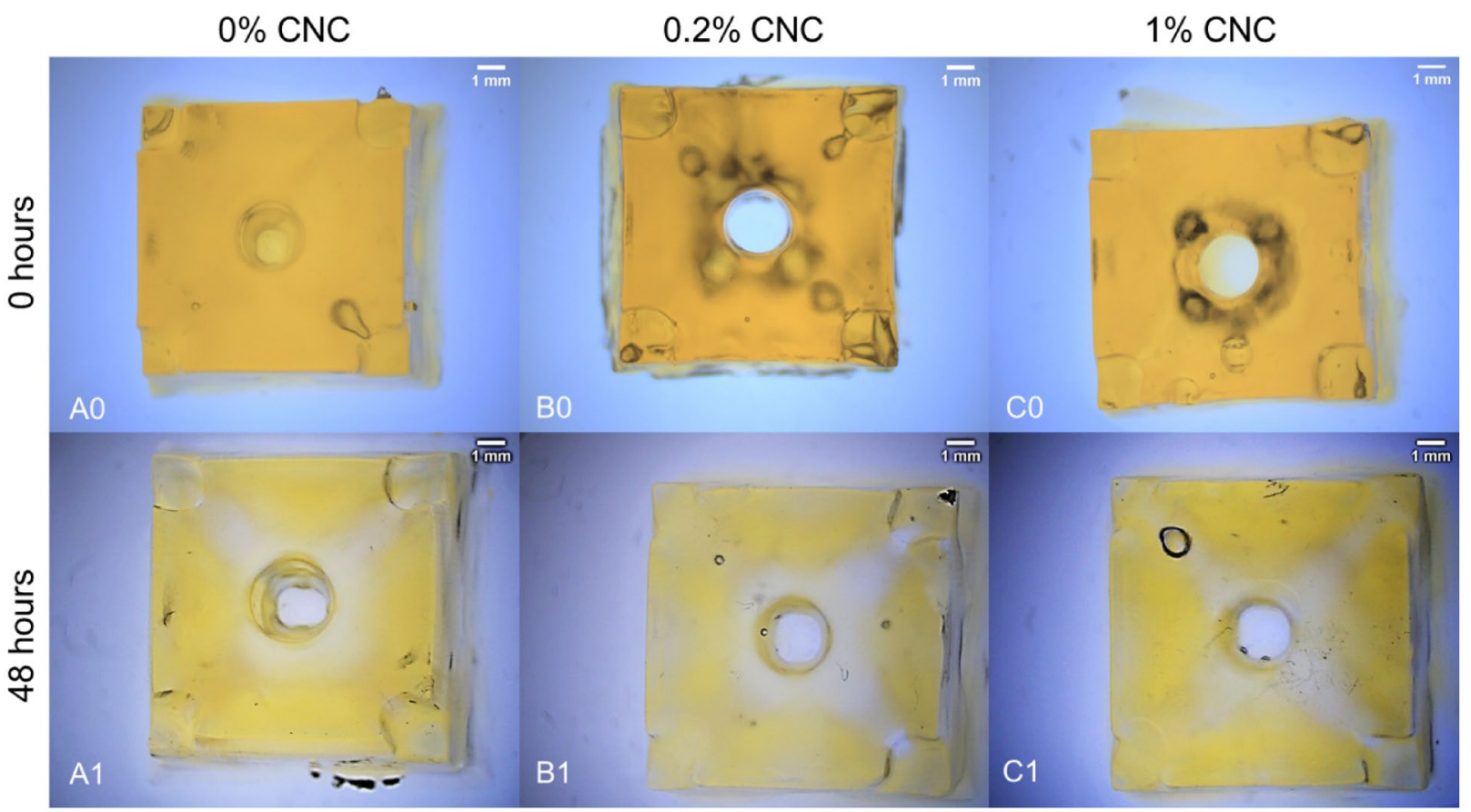

Figure 5: Representative images of Lattice prints before $(0 \mathrm{~h})$ and after swelling ( $48 \mathrm{~h})$. $A=0 \% \mathrm{CNC} ; \mathrm{B}=0.2 \% \mathrm{CNC} ; \mathrm{C}=1 \% \mathrm{CNC}$. At $48 \mathrm{~h}$, the prints become more transparent due to the photoabsorber being released during swelling. 
TABLE 1: Effect of swelling on channel width dimensions as a function of CNC\%.

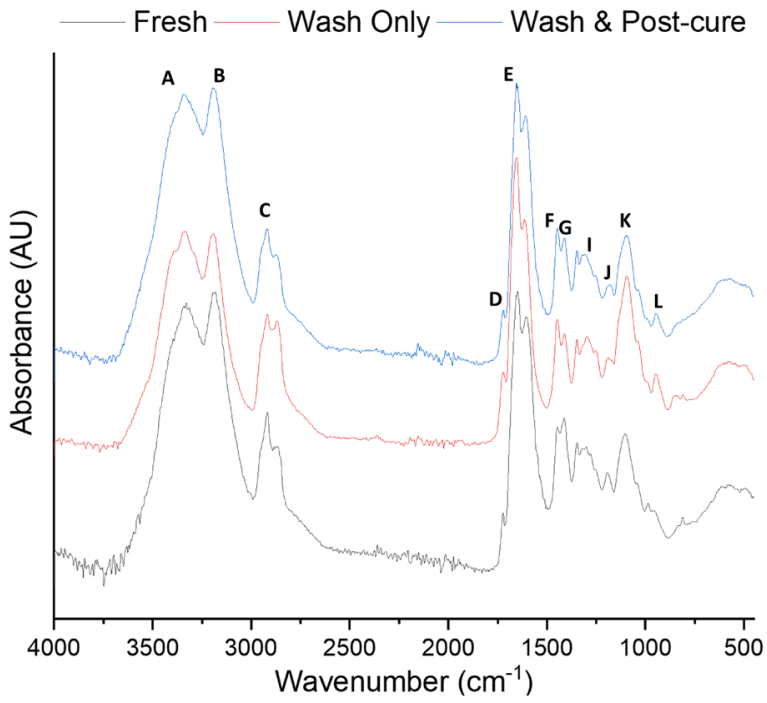

Figure 6: $F T I R$ spectra of $3 \mathrm{D}$ printed AAm/PEGDA ${ }_{700}$ hydrogels with $0 \%$ CNC directly after printing (Fresh), after being soaked in water for $48 \mathrm{~h}$ (Wash Only), and soaked in water for $48 \mathrm{~h}$ and post-cured (Wash and Post-cure). Please refer to Table 2 for a summary of the FTIR bands (A-L).

TABLE 2: Summary of FTIR bands for 3D printed AAm/PEGDA 700 hydrogels.

\begin{tabular}{lllc}
\hline \hline & Bond & \multicolumn{1}{c}{ Type } & $\begin{array}{c}\text { Wavenum- } \\
\text { ber }\left(\mathrm{cm}^{-1}\right)\end{array}$ \\
\hline A & $\mathrm{N}-\mathrm{H}$ & Stretching & 3330 \\
$\mathrm{~B}$ & $\mathrm{O}-\mathrm{H}$ & Water remaining in sample & 3082 \\
$\mathrm{C}$ & $\mathrm{C}-\mathrm{H}$ & Alkyl stretching & 2950 \\
D & $\mathrm{C}=\mathrm{O}$ & Carboxylic acid & 1717 \\
D & $\mathrm{C}=\mathrm{O}$ & Stretching, coupled with N-H bending & 1654 \\
E & $\mathrm{N}-\mathrm{H}$ & N-H bending & 1580 \\
$\mathrm{~F}$ & $\mathrm{C}-\mathrm{N}$ & Stretching primary amide & 1420 \\
$\mathrm{G}$ & $\mathrm{C}-\mathrm{H}$ & Deformation & 1350 \\
I & $\mathrm{N}-\mathrm{H}$ & Bending & 1238 \\
J & $\mathrm{NH} \mathrm{H}_{2}$ & In-plane rocking 1120 & 1100 \\
$\mathrm{~K}$ & $\mathrm{C}-\mathrm{OH}$ & Side group vibrations & 1043 \\
$\mathrm{~L}$ & $\mathrm{C}-\mathrm{H}$ & Out-of-plane rocking & 938 \\
\hline \hline
\end{tabular}

and cross-linkers that are unable to leach out are then photopolymerised by a post-curing step. As acrylamide is cytotoxic as a monomer but biocompatible as a polymer, post-processing by washing and post-curing the prints' is a vital step to ensure minimal cytotoxicity for downstream applications.

\section{Mechanical tests}

Mechanical testing of the 3D printed hydrogels was conducted and compared against their respective Bulk samples, as well as increasing design complexity from solid structures to an internal lattice structure. For tissue engineering applications, design complexity and structural integrity is crucial for supporting organisms to proliferate and grow. 3D printed structures that undergo swelling will also require the ability to withstand internal hydrostatic pressures whilst absorbing water. In this study, samples were tested under mechanical compression until failure was observed. Prior to testing, samples were swollen in DI-water until a plateau in mass was reached. Figure 7 provides a comparison of the mechanical properties of the varying concentrations of $\mathrm{CNC}$ with varying internal structures, compared to their respective Bulk material.

The $0 \%$ CNC 3D printed hydrogels exhibited a higher compressive strength compared to the samples incorporating $\mathrm{CNC}$ at $0.2 \%$ and $1 \%$. A fracture compressive stress of $344 \pm 95 \mathrm{kPa}$ was recorded for $0 \%$ CNC Solid samples compared to $201 \pm 39 \mathrm{kPa}$ and $102 \pm 14 \mathrm{kPa}$ for Solid samples of CNC at $0.2 \%$ and $1 \%$, respectively. At the concentrations tested in this study, the incorporation of CNC results in a decrease in hydrogel crystallinity and therefore a decrease in compressive strength [32]. Change in the internal designs of the hydrogels impacts the compressive stress and deformation significantly. With increasing design complexity, from Solid to Hole to Lattice structures, the compressive strength decreases. For all concentrations of $\mathrm{CNC}$, the lattice structure demonstrated the lowest compressive strength averaging at $29 \mathrm{kPa}$ and deformation values below $20 \%$ for all specimens. This is due to the increase in potential failure points within the lattice when a stress is applied. These results demonstrate the possibility to tune hydrogel designs to achieve a particular elasticity, toughness and strength [42].

In addition, Bulk samples were analysed to evaluate the effect of 3D printing on the mechanical properties of the hydrogels. The Bulk samples are able to withstand larger deformations, with an average of $40 \%$ strain compared to all printed samples, this is likely due to the longer polymer chains holding the internal structure together. However, it was observed that the compressive strength of the Bulk was lower than the $3 \mathrm{D}$ printed Solid structure with $0 \%$ and $0.2 \% \mathrm{CNC}$. This effect has to be further investigated as the three statistical repeats taken during the measurement demonstrate a variability in the compressive stress values for the Solid samples. The overall increase in compressive stress for the $0 \%$ and $0.2 \% \mathrm{CNC}$ Solid Samples is likely due to the compact layer-by-layer build of the 3D prints which act as reinforcement stacks for the Solid 
(a1)

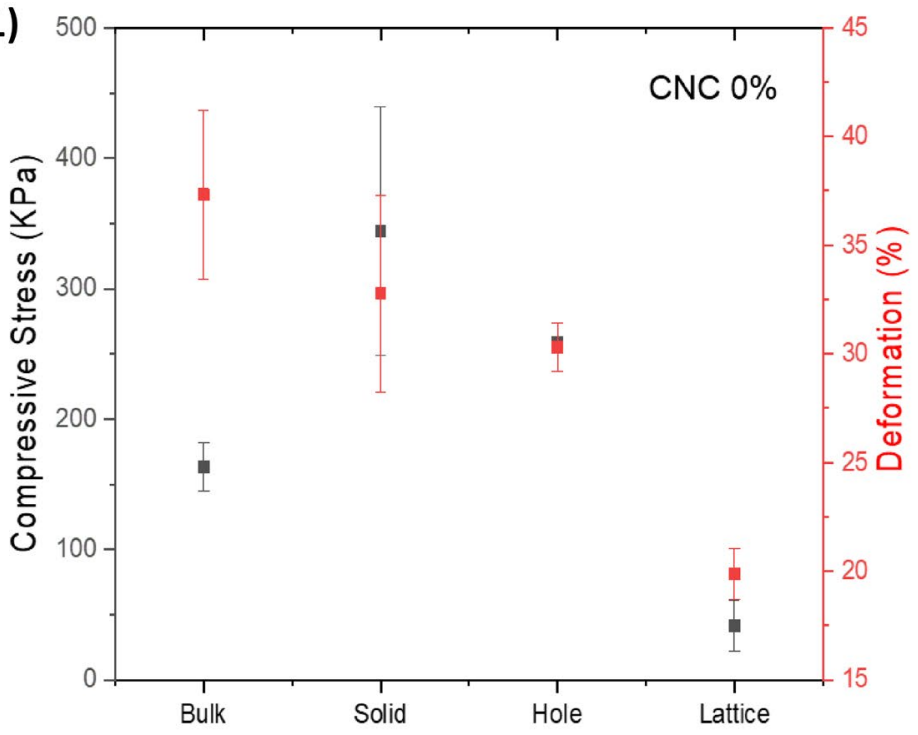

(a2)

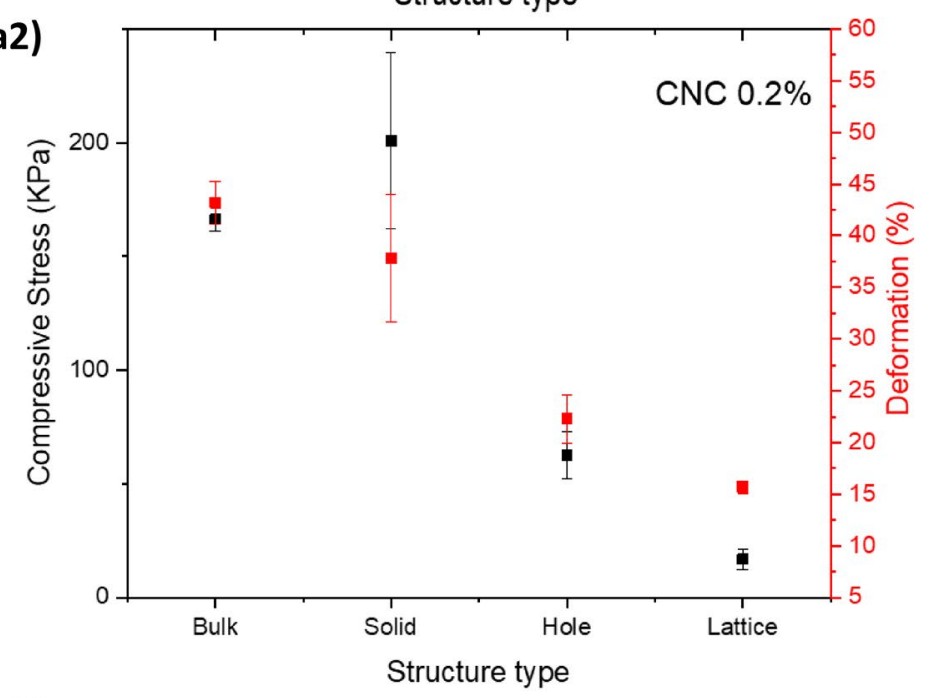

(a3)

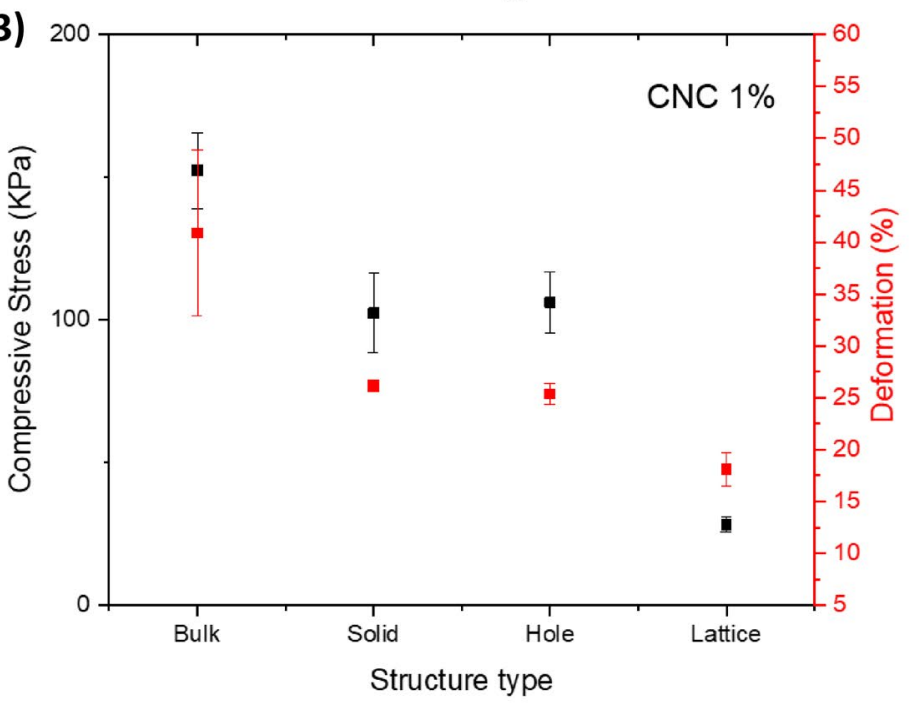

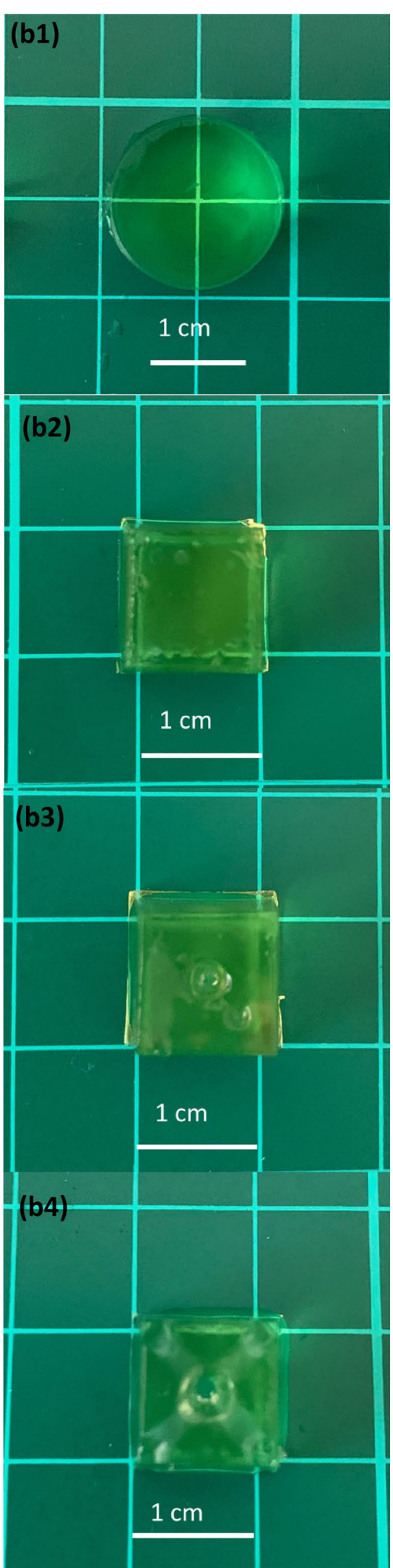

Figure 7: Mechanical testing in compression of Bulk vs. 3D printed hydrogels with solid, hole and lattice structures with CNC concentrations of (a1) $0 \%$, (a2) $0.2 \%$ and (a3) $1 \%$. Images of (b1) Bulk, (b2) solid, (b3) hole and (b4) lattice samples with $0.2 \%$ CNC. 
print as well as local curing with short-pulsed lasers with high energy doses (pulsed-laser polymerisation) compared to a less controlled polymerisation occurring in the Bulk Samples. This effect is reduced at $1 \% \mathrm{CNC}$ due to a likely further decrease in crystallinity [32] and CNC acting as a nanofiller which creates more failure points within the structure. This results in a lower compressive strength compared to its Bulk counterpart at $1 \%$ CNC. This could also be a result of weaker layer adhesion due to light scattering caused by $\mathrm{CNC}$ during the $3 \mathrm{D}$ printing process as well as making the hydrogel less uniform. In addition, $\mathrm{CNC}$ particles may sediment during the $3 \mathrm{D}$ printing process, causing inhomogeneous distribution within the print. As CNC concentration increases, it is also expected that it will hinder the longer polymers from moving freely resulting in decreased free volume
[26]. A decrease in free volume will results in less water uptake and stiffer networks, hence a decrease in deformation values.

\section{Scanning electron microscopy (SEM)}

To examine the internal hydrogel structure, SEM was used to determine the presence of $\mathrm{CNC}$ polymer webs and CNC aggregates as well as a qualitative analysis on porosity within the hydrogel structure. All samples were frozen to $-80^{\circ} \mathrm{C}$ and then freeze-dried for $24 \mathrm{~h}$ prior to imaging. Figure 8 shows the differences between cross-section of the Bulk hydrogels and Solid $3 \mathrm{D}$ printed hydrogels. CNC polymer webs become more evident with increasing $\mathrm{CNC}$ concentration. CNC polymer webs consist of polymers that become entangled with $\mathrm{CNC}$ during

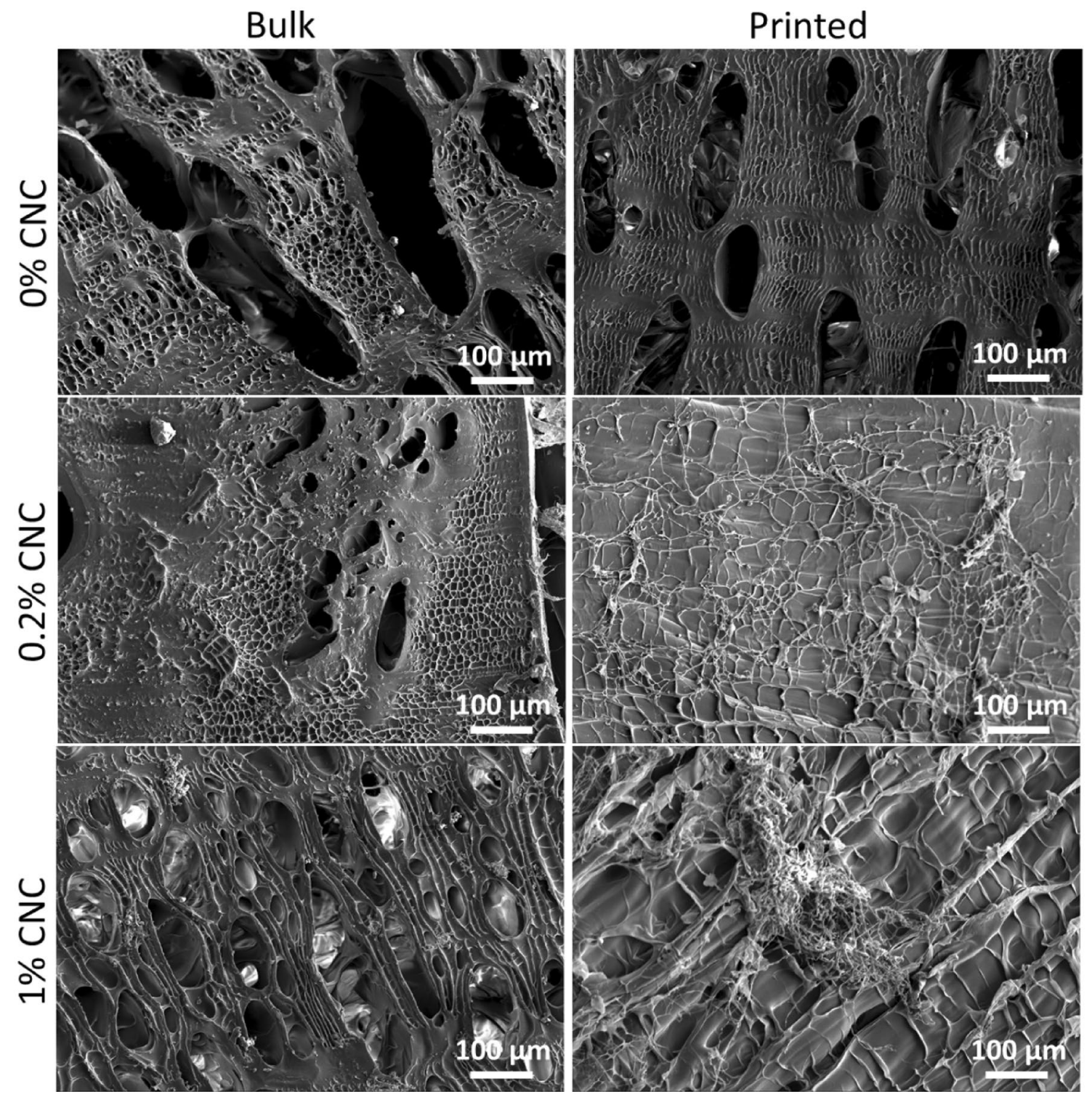

Figure 8: SEM analysis of Bulk vs. 3D printed Solid hydrogels for $0 \%, 0.2 \%$ and $1 \%$ CNC. All images were taken at $\times 150$ magnification. 
photopolymerisation. For 3D printed specimens this behaviour propagates along the internal structure in the $x-y$ plane during printing process. This can be seen through the SEM images of the cross-sections of the $3 \mathrm{D}$ printed hydrogels (Fig. 8). This is more prominent between layers in the $3 \mathrm{D}$ printed structures relative to the Bulk samples where the $\mathrm{CNC}$ presence would be integrated into the overall polymer network. This is due to the inherent photopolymerisation techniques used for both structures. For SLA, the laser shines for a defined number of seconds per layer and stops before moving to the next layer. The pause in between each layer causes the photopolymerisation to stop, and therefore the chains are limited in length due to this on/ off process. The photopolymerisation process starts again once the next layer is being formed. Alongside this, the local area where the photopolymerisation occurs is displaced with fresh resin as the platform moves up and down, which keeps the resin well-mixed and homogenous. In contrast, Bulk photopolymerisation occurs for a set reaction time with a light shining across the entire Bulk, without pausing or displacing the local volume, resulting in longer molecular weight chains. However, the centre of the Bulks volume will have a different light and energy exposure compared to the surface. Therefore, the Bulk samples require a longer reaction time to ensure a complete photopolymerisation occurs throughout the entire volume.

The SEM images also provide evidence that sonification of $\mathrm{CNC}$ prior to printing was sufficient to ensure homogenous dispersion as no CNC aggregates were observed. Finally, compared to the Bulk samples, the 3D printed hydrogels show a more homogenous porous structure due to the repetitive layerby-layer photopolymerisation process which provides consistent illumination and energy distribution in a localised region. The differences between the cross-sectional structures at the microscale of the 3D printed samples compared to Bulk samples provide further insight into the physical properties of these hydrogels. Further studies such as mercury porosimetry can be carried out for further investigation on total pore volume between the Bulk and Solid sample.

\section{Conclusion}

In this work, we have successfully developed an optimisation process for 3D printing hydrogel structures using a Stereolithography $3 \mathrm{D}$ printing technique. A photocurable formulation has been demonstrated using commercially available monomers. Control on the mechanical properties and multi-dimensional pore structure is achieved by varying structure design and $\mathrm{CNC}$ concentration. The incorporation of $\mathrm{CNC}$ at low percentages results in a decrease of the overall mechanical properties of the structure but improves shape fidelity and results in a reduction in dimensional changes after swelling. Also, the presence of CNC polymer webs influences surface topography by providing it with extra roughness. This could be advantageous for cell attachment and proliferation and therefore beneficial for applications in tissue engineering. The versatility of the hydrogels in this study could also be advantageous across several other fields including hydrogels for soilless cultivation and soft robotics, where rapid prototyping can be used to identify optimal designs. Exploiting the benefits of hydrogels and combining them with additive manufacturing techniques can lead to the fabrication of a bionic system. Such a system could provide an enhanced habitat for complex organisms to thrive by tuning the design and chemical properties of the system.

\section{Materials and methods}

\section{Materials}

The following reagents were purchased from Sigma-Aldrich (Dorset, UK) and used as received unless stated otherwise: acrylamide (AAm; $\geq 99 \%$ ), poly (ethylene glycol) diacrylate (PEGDA 700; average Mn 700), lithium phenyl-2,4,6-trimethylbenzoylphosphinate (LAP; $\geq 95 \%$ ), 1-phenylazo-2-naphthol-6,8-disulfonic acid disodium salt (Orange G). Cellulose Nanocrystal (CNC; diameter: 10-20 nm, length: 300-900 nm) was purchased from Nanografi and used as received.

\section{CAD design and 3D printing software}

Test specimens consisted of $1 \mathrm{~cm}^{3}$ cubes designed using Fusion 360 (Autodesk, USA). The designs consisted of a solid cube (Solid), a cube with a single channel (Hole) and a cube with interconnected channels (Lattice). The three designs were saved as STL files and uploaded to the Formlabs printing software (Preform) without the addition of any support structures. The settings used were 'Clear Laser V.4' and a layer build height of $100 \mu \mathrm{m}$. The STL files were then uploaded directly from Preform to a Form 2 printer.

Discs and minimum channel structures were also designed to evaluate the printability and highest possible printable resolution for the formulation in this study. The dimension of the discs was $1 \mathrm{~cm}$ in diameter, whilst the minimum channel structures were 0.2. $0.30 .4,0.5$ and $0.75,1,1.25$ and $1.5 \mathrm{~mm}$ in width on the top surface. The channel structures were printed with a taper angle to facilitate the creation of a channel with dimensions on the top surface as mentioned above. The taper angle mitigates the effect of light penetrating from previous layers and hence causing overcuring of the channel.

\section{Resin formulation and 3D printing}

Based on a final total resin volume of $250 \mathrm{~g}, 15 \mathrm{w} / \mathrm{w} \% \mathrm{PEGDA}_{700}$ and $5 \mathrm{w} / \mathrm{w} \%$ AAm monomers were added to a clean $250 \mathrm{ml}$ amber bottle. $200 \mathrm{~g}$ of deionised water was added to the mixture and left to stir using a magnetic stirring plate for $30 \mathrm{~min}$ until fully 
dissolved. Next, $1 \%$ LAP and $0.08 \%$ Orange G (relative to monomer mass) were added to the mixture and left to stir for another $30 \mathrm{~min}$ until fully dissolved. Once the formulation was ready it was loaded into a Formlabs Form2 SLA printer (405 nm light source) using the 'Open Mode' setting suitable for 3rd party resins. The STL file consisting of the three cube structures (Solid, Hole and Lattice) was uploaded to the Form2 printer and left to print. Once the print was complete, the build platform was detached from the printer, and samples were rinsed with DI-water until all excess resin was removed. The prints were detached from the build platform and rinsed again with DI-water. Detached samples were post-cured for $3 \mathrm{~min}$ using the Formlabs Form Cure postcuring station (405 $\mathrm{nm}$ light source) to polymerise any unreacted monomers. Post-cured samples were then prepared for swelling studies, optical, chemical, and mechanical analysis.

The above was repeated for samples containing $0.2 \%$ and $1 \%$ CNC (relative to monomer mass). CNC was added after LAP and Orange G. The formulation was sonicated for $30 \mathrm{~min}$ in a sonication bath until the CNC was homogeneously dispersed. The same settings and post printing procedure discussed above were used for the CNC formulations.

Bulk hydrogels were synthesised using the three formulations above to be used for comparison during mechanical analysis. Bulk hydrogels were prepared by curing the resin in a 24 cell well plate, using the Form Cure, to form cylindrical samples of $1.5 \mathrm{~cm}$ in diameter, and $1.5 \mathrm{~cm}$ in height. The Bulk hydrogels were swollen for $48 \mathrm{~h}$ prior to analysis.

\section{Optical imaging and swelling studies}

Freshly prepared samples were imaged using a UltraZOOM-3 GX Optical Microscope to examine the resolution of the holes relative to the CAD design, prior to swelling in water. The samples (three repeats per design) were weighed at time $0 \mathrm{~h}$, swollen in DI-water for $48 \mathrm{~h}$ and weighed again to calculate the swelling ratio for each of the three structures (Solid, Hole and Lattice). The samples were imaged again to study changes in hole diameters post-swelling.

\section{Fourier-transform infrared spectroscopy (FTIR)}

Three samples containing $0 \%$ CNC were investigated using a Nicolet iS10 Thermo Scientific FTIR. The first sample was not washed nor post-cured, the second sample was washed in DI-water, and the third sample was both washed and postcured. The three samples were dried in a $60^{\circ} \mathrm{C}$ oven for $48 \mathrm{~h}$ then ground to a fine powder for FTIR analysis. Samples were scanned 32 times per run with a resolution of 8 in a wavenumber region of $4000-400 \mathrm{~cm}^{-1}$.

\section{Mechanical testing}

Swollen samples were used for mechanical analysis in compression, using a Zwick Roell z2.5 machine fitted with a load cell of $10 \mathrm{kN}$, and a strain rate of $1.5 \mathrm{~mm} / \mathrm{min}$. Five repeats per sample structure (Solid, Hole and Lattice) were tested and compared to corresponding Bulk hydrogel. Mechanical testing was conducted on all swollen samples until mechanical failure of the material was achieved. Failure of the material was considered to be the first sign of breakage whilst under strain. During sample preparation and removal from the printing build plate care was taken to ensure samples had flat bottom and top surfaces to ensure an even distribution of force during testing.

\section{Scanning electron microscopy (SEM)}

Both Bulk and 3D printed swollen hydrogel samples were cut into cube shaped pieces $(5 \mathrm{~mm} \times 5 \mathrm{~mm} \times 5 \mathrm{~mm})$ to examine cross-sections of their internal structures. The cut samples were placed in $\mathrm{a}-80^{\circ} \mathrm{C}$ freezer overnight to avoid structural collapse during freeze drying. Samples were then freeze-dried for $24 \mathrm{~h}$. Prior to SEM imaging, freeze-dried samples were sputter coated with gold for $2 \mathrm{~min}$ at $20 \mathrm{~mA}$ to form a $15 \mathrm{~nm}$ layer. Next, silver was dissolved in acetone and used to coat one edge of each sample to avoid charging whilst imaging. Samples were imaged using the JEOL 6010LA microscope in secondary electron mode at $8-12 \mathrm{kV}$ with a working distance of $8-13 \mathrm{~mm}$.

\section{Author contributions}

AAM and LMK contributed equally to the study conception, design, analysis, data collection and writing. All authors read and approved the final manuscript.

\section{Funding}

This work was supported by The Leverhulme Trust's 'Leverhulme Doctoral Scholarship Programme' (Grant Number: DS-2017-087) and the Engineering and Physical Research Council (Grant number: EP/L015498/1), and the Imperial College London President's Excellence Fund for Frontier Research.

\section{Data availability}

The data that support the tables within this paper and other findings of this study are available from the corresponding author upon reasonable request. 


\section{Declarations}

Conflict of interest The authors declare no conflict of interest with regard to the submitted manuscript.

\section{Supplementary Information}

The online version contains supplementary material available at https://doi.org/10.1557/s43578-021-00411-2.

\section{Open Access}

This article is licensed under a Creative Commons Attribution 4.0 International License, which permits use, sharing, adaptation, distribution and reproduction in any medium or format, as long as you give appropriate credit to the original author(s) and the source, provide a link to the Creative Commons licence, and indicate if changes were made. The images or other third party material in this article are included in the article's Creative Commons licence, unless indicated otherwise in a credit line to the material. If material is not included in the article's Creative Commons licence and your intended use is not permitted by statutory regulation or exceeds the permitted use, you will need to obtain permission directly from the copyright holder. To view a copy of this licence, visit http://creativecommons.org/licenses/by/4.0/.

\section{References}

1. A.S. Hoffman, Hydrogels for biomedical applications. Adv. Drug Deliv. Rev. 64, 18 (2012)

2. Y.S. Zhang, A. Khademhosseini, Advances in engineering hydrogels. Science 356(6337), eaaf3627 (2017)

3. M.W. Tibbitt, K.S. Anseth, Hydrogels as extracellular matrix mimics for 3D cell culture. Biotechnol. Bioeng. 103(4), 655 (2009)

4. B.V. Slaughter, S.S. Khurshid, O.Z. Fisher, A. Khademhosseini, N.A. Peppas, Hydrogels in regenerative medicine. Adv. Mater. 21(32-33), 3307 (2009)

5. H. Omidian, K. Park, Introduction to hydrogels, in Biomedical Applications of Hydrogels Handbook. ed. by R.M. Ottenbrite, K. Park, T. Okano (Springer, New York, 2010), p. 1

6. D.S. Benoit, M.P. Schwartz, A.R. Durney, K.S. Anseth, Small functional groups for controlled differentiation of hydrogelencapsulated human mesenchymal stem cells. Nat. Mater. 7(10), 816 (2008)

7. T.R. Hoare, D.S. Kohane, Hydrogels in drug delivery: Progress and challenges. Polymer 49(8), 1993 (2008)

8. J.P. Gong, Why are double network hydrogels so tough? Soft Matter 6(12), 2583 (2010)

9. Q. Chen, H. Chen, L. Zhu, J. Zheng, Fundamentals of double network hydrogels. J. Mater. Chem. B 3(18), 3654 (2015)
10. J.P. Gong, Y. Katsuyama, T. Kurokawa, Y. Osada, Double-network hydrogels with extremely high mechanical strength. Adv. Mater. 15(14), 1155 (2003)

11. A.A. Mohammed, J. Aviles Milan, S. Li, J.J. Chung, M.M. Stevens, T.K. Georgiou, J.R. Jones, Open vessel free radical photopolymerization of double network gels for biomaterial applications using glucose oxidase. J. Mater. Chem. B 7(25), 4030 (2019)

12. M.E. Villanueva, A.M.D.R. Diez, J.A. González, C.J. Pérez, M. Orrego, L. Piehl, S. Teves, G.J. Copello, Antimicrobial activity of starch hydrogel incorporated with copper nanoparticles. ACS Appl. Mater. Interfaces 8(25), 16280 (2016)

13. C.-W. Chang, A. van Spreeuwel, C. Zhang, S. Varghese, PEG/ clay nanocomposite hydrogel: A mechanically robust tissue engineering scaffold. Soft Matter 6(20), 5157 (2010)

14. A.A. Mohammed, A. Pinna, S. Li, T. Sang, J.R. Jones, Autocatalytic redox polymerisation using nanoceria and glucose oxidase for double network hydrogels. J. Mater. Chem. B 8(14), $2834(2020)$

15. C. Zhou, Q. Wu, Y. Yue, Q. Zhang, Application of rod-shaped cellulose nanocrystals in polyacrylamide hydrogels. J. Colloid Interface Sci. 353(1), 116 (2011)

16. T. Abitbol, T. Johnstone, T.M. Quinn, D.G. Gray, Reinforcement with cellulose nanocrystals of poly(vinyl alcohol) hydrogels prepared by cyclic freezing and thawing. Soft Matter 7(6), $2373(2011)$

17. Z. Deng, T. Hu, Q. Lei, J. He, P.X. Ma, B. Guo, Stimuliresponsive conductive nanocomposite hydrogels with high stretchability, self-healing, adhesiveness, and 3D printability for human motion sensing. ACS Appl. Mater. Interfaces 11(7), 6796 (2019)

18. A. Motealleh, P. Dorri, A.H. Schäfer, N.S. Kehr, 3D bioprinting of triphasic nanocomposite hydrogels and scaffolds for cell adhesion and migration. Biofabrication 11(3), 035022 (2019)

19. N. Das: Preparation methods and properties of hydrogel. (City, 2013).

20. A.M. Mathur, S.K. Moorjani, A.B. Scranton, Methods for synthesis of hydrogel networks: A review. J. Macromol. Sci. C 36(2), 405 (1996)

21. J.A. Burdick, W.L. Murphy, Moving from static to dynamic complexity in hydrogel design. Nat. Commun. 3(1), 1269 (2012)

22. J. Warner, P. Soman, W. Zhu, M. Tom, S. Chen, Design and 3D printing of hydrogel scaffolds with fractal geometries. ACS Biomater. Sci. Eng. 2(10), 1763 (2016)

23. L.M. Kalossaka, G. Sena, L.M.C. Barter, C. Myant, Review: 3D printing hydrogels for the fabrication of soilless cultivation substrates. Appl. Mater. Today 24, 101088 (2021)

24. A.G.P. Kottapalli, M. Asadnia, J.M. Miao and M.S. Triantafyllou: Electrospun nanofibrils encapsulated in hydrogel cupula for biomimetic MEMS flow sensor development, in 2013 IEEE 26th International Conference on Micro Electro Mechanical Systems (MEMS), (City, 2013), pp. 25. 
25. A.G.P. Kottapalli, M. Bora, M. Asadnia, J. Miao, S.S. Venkatraman, M. Triantafyllou, Nanofibril scaffold assisted MEMS artificial hydrogel neuromasts for enhanced sensitivity flow sensing. Sci. Rep. 6(1), 19336 (2016)

26. N.B. Palaganas, J.D. Mangadlao, A.C.C. de Leon, J.O. Palaganas, K.D. Pangilinan, Y.J. Lee, R.C. Advincula, 3D printing of photocurable cellulose nanocrystal composite for fabrication of complex architectures via stereolithography. ACS Appl. Mater. Interfaces 9(39), 34314 (2017)

27. Y. Jiang, J. Zhou, H. Shi, G. Zhao, Q. Zhang, C. Feng, X. Xv, Preparation of cellulose nanocrystal/oxidized dextran/gelatin (CNC/OD/GEL) hydrogels and fabrication of a CNC/OD/GEL scaffold by 3D printing. J. Mater. Sci. 55(6), 2618 (2020)

28. L. Shuai, Z.H. Guo, P. Zhang, J. Wan, X. Pu, Z.L. Wang, Stretchable, self-healing, conductive hydrogel fibers for strain sensing and triboelectric energy-harvesting smart textiles. Nano Energy 78, 105389 (2020)

29. S. Wei, G. Qu, G. Luo, Y. Huang, H. Zhang, X. Zhou, L. Wang, Z. Liu, T. Kong, Scalable and automated fabrication of conductive tough-hydrogel microfibers with ultrastretchability, 3D printability, and stress sensitivity. ACS Appl. Mater. Interfaces 10(13), 11204 (2018)

30. G.A. Appuhamillage, N. Chartrain, V. Meenakshisundaram, K.D. Feller, C.B. Williams, T.E. Long, 110th anniversary: Vat photopolymerization-based additive manufacturing: Current trends and future directions in materials design. Ind. Eng. Chem. Res. 58(33), 15109 (2019)

31. I. Gibson, D. Rosen, B. Stucker, Vat Photopolymerization Processes, in Additive manufacturing technologies: $3 D$ printing, rapid prototyping, and direct digital manufacturing. ed. by I. Gibson, D. Rosen, B. Stucker (Springer, New York, 2015), p. 63

32. S. Butylina, S. Geng, K. Laatikainen, K. Oksman, Cellulose nanocomposite hydrogels: From formulation to material properties. Front. Chem. 8(655), 1 (2020)
33. J. Wang, A. Chiappone, I. Roppolo, F. Shao, E. Fantino, M. Lorusso, D. Rentsch, K. Dietliker, C.F. Pirri, H. Grützmacher, All-in-one cellulose nanocrystals for 3D printing of nanocomposite hydrogels. Angew. Chem. Int. Ed. 57(9), 2353 (2018)

34. S. Sultan, A.P. Mathew, 3D printed scaffolds with gradient porosity based on a cellulose nanocrystal hydrogel. Nanoscale 10(9), 4421 (2018)

35. D. Kam, A. Braner, A. Abouzglo, L. Larush, A. Chiappone, O. Shoseyov, S. Magdassi, 3D printing of cellulose nanocrystalloaded hydrogels through rapid fixation by photopolymerization. Langmuir 37(21), 6451 (2021)

36. B. Zhang, S. Li, H. Hingorani, A. Serjouei, L. Larush, A.A. Pawar, W.H. Goh, A.H. Sakhaei, M. Hashimoto, K. Kowsari, S. Magdassi, Q. Ge, Highly stretchable hydrogels for UV curing based high-resolution multimaterial 3D printing. J. Mater. Chem. B 6(20), 3246 (2018)

37. B. Webb, B.J. Doyle, Parameter optimization for 3D bioprinting of hydrogels. Bioprinting 8, 8 (2017)

38. Y. Ide, S. Nayar, H. Logan, B. Gallagher, J. Wolfaardt, The effect of the angle of acuteness of additive manufactured models and the direction of printing on the dimensional fidelity: clinical implications. Odontology 105(1), 108 (2017)

39. M. Braian, R. Jimbo, A. Wennerberg, Production tolerance of additive manufactured polymeric objects for clinical applications. Dental Mater. 32(7), 853 (2016)

40. J. Yang, C.-R. Han, J.-F. Duan, F. Xu, R.-C. Sun, Mechanical and viscoelastic properties of cellulose nanocrystals reinforced poly(ethylene glycol) nanocomposite hydrogels. ACS Appl. Mater. Interfaces 5(8), 3199 (2013)

41. A. Mohammed: Double network hydrogels for cartilage repair and their nanocomposite structure (2017).

42. S. Ma, B. Yu, X. Pei, F. Zhou, Structural hydrogels. Polymer 98, 516 (2016) 